\title{
Semantic abilities and narration in aphasia in Greek
}

Fani Nasika

Hellenic Open University, Greece

https://doi.org/10.36505/ExLing-2019/10/0037/000399

\begin{abstract}
This paper aims to present the findings of clinical linguistic analysis on narrative speech in aphasia. Aphasia is an acquired language disorder caused by brain lesions and it can be distinguished into non-fluent and fluent aphasia. Both categories show linguistic deficits affecting language production and/or comprehension. Aphasic narrative speech is considered particularly impaired in aphasia in respect of morphosyntax and semantics (Goodglass, Kaplan, Barresi 2001). Thus, in this study, the semantic abilities of four aphasic Greek speakers, two with non-fluent and two with fluent aphasia, were examined during storytelling. The samples were analyzed semantically with the use of PRISM (Crystal 1992). The results showed the semantic abilities of the participants both at a lexical level and at thematic structure level.
\end{abstract}

Key words: PRISM, semantic analysis, aphasia, Greek.

\section{Theoretical background}

Aphasia is an acquired language disorder which can affect skills related to language expression and/or comprehension, thus highly compromising communication (Goodglass, Kaplan, Barresi 2001), while it is caused by lesions in the brain, following a cardio-vascular accident (CVA), a tumour, a traumatic brain injury (TBI) or other neurological causes. These lesions should be located in the language-dominant hemisphere. Moreover, aphasia can be distinguished into two main aphasia types: the non-fluent and the fluent aphasia. This distinction has been based on the fact that the most prominent feature differentiating these two types of aphasia is fluency (Goodglass, Kaplan, Barresi 2001).

With reference to non-fluent aphasia, following Goodglass, Kaplan and Barresi (2001), it is characterized by non-fluent speech production, reduced or ungrammatical sequences, pauses, and difficulties with articulation, inflection and word retrieval. In relation to fluent aphasia, individuals have word finding difficulties, especially with nouns, and their comprehension is impaired. Their errors include omissions and substitutions as well as paraphasias. Finally, they may present with difficulties in thematic role assignment both in production and comprehension (Caramazza, Miceli 1991).

In this respect, it seems that both individuals with non-fluent and fluent aphasia face difficulties with narrative speech, although these difficulties may be

ExLing 2019: Proceedings of 10 $0^{\text {th }}$ International Conference of Experimental Linguistics, 25-27 September 2019, Lisbon, Portugal 
of different nature (Goodglass, Kaplan, Baresi 2001), thus expecting more morphosyntactic errors in non-fluent aphasia and more semantic errors in fluent aphasia. A tool for profiling these abilities is PRISM (Crystal 1992). PRISM comprises two separate procedures: PRISM-L, which examines the relationship between semantics and the mental lexicon, and PRISM-G, which examines the relationship between semantics and grammar (i.e., thematic-role structures). In the present study, PRISM-L and PRISM-G were used to profile the semantic abilities of Greek-speaking individuals with aphasia during the narration of a story.

\section{Methodology}

\section{Sample}

The narrative speech samples were collected from four participants, two with non-fluent aphasia and two with fluent aphasia $(M=56.5 ; S D=19.8$; range $=$ 39-77). There were three male and one female participant. All participants had suffered a CVA at least 6 months before participating in this study. In order for them to participate, they were assessed with the Greek standardized adaptation of the Boston Diagnostic Aphasia Examination (BDAE) (Goodglass, Kaplan 1972; for the Greek adaptation: Papathanasiou, Feidantsi, Katsantoni, Panagiotopoulou, Malefaki 2004).

\section{Material and procedure}

The speech samples were collected through story-telling, with the use of a Cinderella story booklet with pictures (Berndt, Wayland, Rochon, Saffran, Schwartz 2000). The collected samples were analyzed with the use PRISM (Crystal 1992), which was adapted into Greek (Nasika, Afantenou, Kremmyda 2017). More specifically, the samples were analyzed at a lexical level with PRISM-L and at a thematic structure level with PRISM-G.

\section{Results}

Regarding participant 01, he was non-fluent and, in his narration, he used words from 24 out of 61 semantic fields, namely man, clothing, moving, making/doing, sight, language, quantity, time, etc. Of these semantic fields, the ones with the smallest lexical range were making/doing, happening, sight, and place. Concerning PRISM-G, he produced mainly clauses of two $(62 \%)$ or three $(22 \%)$ semantic elements, which places him in Stages II and III. The most frequent combination in Stage II was that of a dynamic verb with a theme element $(65 \%)$ while in Stage III the most frequent combination was that of a dynamic verb with a theme and a temporal, locative or other element $(55 \%)$. Very often, he omitted the Actor of the action or the Experiencer.

Referring to participant 02 , he was non-fluent and he produced words from 20 out of 61 semantic fields while not producing words from the last 12 semantic fields of PRISM-L, which correspond to later stages of lexical 
development. The semantic fields covered were mostly man, body, clothing, moving, tools, animals, food, make/do, etc. Of these semantic fields, the ones with the smallest lexical range were body and clothing. Regarding PRISM-G, participant 02 produced clauses with two $(71 \%)$ or three $(29 \%)$ semantic elements, which places him in Stages II and III. The main thematic structure used was that of a dynamic verb with a theme element. The Actor element was used only once.

As for participant 03, she had fluent aphasia. She used words from 14 out of 61 semantic fields, namely man, clothing, moving, making/doing, happening, place, time, etc. Of these semantic fields, the one with the smallest lexical range was body. Concerning PRISM-G, she produced mainly clauses of one $(32 \%)$ or two $(58 \%)$ semantic elements, which places her in Stages I and II. The most frequent structure in Stage I was that of a dynamic verb (83\%) while in Stage II the most frequent combination was that of a dynamic verb with a theme (45\%). Very often, she omitted the Actor of the action.

Concerning participant 04 , he also had fluent aphasia. He produced words from 22 out of 61 semantic fields, namely man, clothing, moving, making/doing, happening, feeling, quantity, place, time, etc. Of these semantic fields, the ones with the smallest lexical range were man and clothing. Concerning PRISM-G, he produced mainly clauses of two (70\%) or three $(18 \%)$ semantic elements, which places him in Stages II and III. The most frequent structure in Stage II was that of a dynamic verb with a theme $(30 \%)$ while in Stage III the most frequent combination was that of an actor + dynamic verb + theme $(33 \%)$.

\section{Conclusions}

The profiling of the semantic abilities of the four participants with the use of PRISM showed similarities irrespective of the type of aphasia. More specifically, regarding PRISM-L, it was shown that all participants used on average 33\% of the available semantic fields while there was variation concerning the lexical range of the fields used. In relation to PRISM-G, most participants used mainly two and three-element thematic structures, corresponding to Stages II and III, while one participant with fluent aphasia used mainly structures from Stage I and Stage II. The thematic structure mostly used was that of a dynamic verb + theme. It should be noted that the Actor element was frequently omitted. However, this omission does not lead to an ungrammatical structure in Greek but it may have consequences for the cohesion and the coherence of the narration.

Overall, it can be seen that the participants used a small range of semantic fields while they used simple thematic structures which correspond to early developmental stages. Thus, it seems that their semantic abilities are compromised and they bear certain similarities which should be further studied. In addition, it can be seen that PRISM is a useful tool for profiling the semantic 
abilities of individuals with aphasia, thus, providing information for clinical assessment and intervention.

\section{References}

Berndt, R.S., Wayland, S., Rochon, E., Saffran, E., Schwartz, M. 2000. Quantitative production analysis. Training manual. Hove, Psychology Press.

Caramazza, A., Miceli, G. 1991. Selective Impairment of Thematic Role Assignment in Sentence Processing. Brain and Language, 41, 402-436.

Crystal, D. 1992. Profiling linguistic disability. London, Whurr Publishers.

Goodglass, H., Kaplan, E. 1972. The Assessment of Aphasia and Related Disorders. London, Henry Kimpton Publishers.

Goodglass, H., Kaplan, E., Barresi, B. 2001. The Assessment of Aphasia and Related Disorders. $3^{\text {rd }}$ Edition. Lippincott Williams \& Wilkins.

Nasika, F., Afantenou, K., Kremmyda, G. 2017. Using PRISM to profile semantic ability in Greek. In Botinis, A. (ed.), Proc. $8^{\text {th }}$ Tutorial \& Research Workshop on Experimental Linguistics, 77-80, Heraklion, Crete, Greece.

Papathanasiou, I., Feidatsi, M., Katsantoni, M., Panagiotopoulou, E., Malefaki, S. 2004 The validation of Boston Diagnostic Aphasia Examination (BDAE-3) in Greek. Paper presented at the 26th World Congress of the International Association of Logopaedics and Phonetics, 29 August-2 September 2004, Brisbane Australia. 